\title{
Flavour-selective localization in interacting lattice fermions via SU(N) symmetry breaking
}

\section{Daniele Tusi}

LENS - European Laboratory of Non-Linear Spectroscopy

Lorenzo Franchi

University of Florence

Lorenzo Livi

University of Florence

Karla Baumann

SISSA - Scuola Internazionale Studi Superiori

Daniel Benedicto-Orenes

ICFO https://orcid.org/0000-0002-8098-012X

Lorenzo Del Re

Università di Roma

Rafael Barfknecht

CNR-INO https://orcid.org/0000-0002-3883-8437

Tianwei Zhou

https://orcid.org/0000-0001-5830-6326

Massimo Inguscio

LENS and Università degli Studi di Firenze

Giacomo Cappellini

LENS

Massimo Capone

SISSA - Scuola Internazionale Superiore di Studi Avanzati

Jacopo Catani

INO-CNR \& LENS - University of Florence

Leonardo Fallani ( $\square$ fallani@lens.unifi.it)

University of Florence https://orcid.org/0000-0001-5327-3254

\section{Article}

Keywords: quantum physics, superconductors, topological insulators

Posted Date: June 9th, 2021 
DOl: https://doi.org/10.21203/rs.3.rs-543907/v1

License: (c) (1) This work is licensed under a Creative Commons Attribution 4.0 International License. Read Full License

Version of Record: A version of this preprint was published at Nature Physics on September 22nd, 2022. See the published version at https://doi.org/10.1038/s41567-022-01726-5. 


\title{
Flavour-selective localization in interacting lattice fermions via $\mathrm{SU}(\mathrm{N})$ symmetry breaking
}

\author{
D. Tusi ${ }^{1}$, L. Franchi ${ }^{2}$, L. F. Livi ${ }^{2}$, K. Baumann ${ }^{3,4}$, D. Benedicto Orenes ${ }^{5,6}$, L. Del Re ${ }^{7}$, R. E. Barfknecht ${ }^{5}$ \\ T. Zhou ${ }^{2}$, M. Inguscio ${ }^{8,1,5}$, G. Cappellini ${ }^{5,1}$, M. Capone ${ }^{3,9}$, J. Catani ${ }^{5,1}$, L. Fallani ${ }^{2,1,5,10}$ \\ ${ }^{1}$ LENS European Laboratory for Nonlinear Spectroscopy (Sesto Fiorentino, Italy) \\ 2 Department of Physics and Astronomy, University of Florence (Sesto Fiorentino, Italy) \\ ${ }^{3}$ SISSA Scuola Internazionale Superiore di Studi Avanzati (Trieste, Italy) \\ ${ }^{4}$ Laboratoire de Physique et Etude des Matériaux, UMR8213 CNRS/ESPCI/UPMC (Paris, France) \\ ${ }^{5}$ CNR-INO Istituto Nazionale di Ottica del Consiglio Nazionale delle Ricerche (Sesto Fiorentino, Italy) \\ ${ }^{6}$ ICFO - Institut de Ciències Fotòniques, The Barcelona Institute of Science and Technology (Castelldefels, Spain) \\ ${ }^{7}$ Department of Physics, Georgetown University, 37th and O Sts., NW, Washington, DC 20057, USA \\ ${ }^{8}$ Department of Engineering, Campus Bio-Medico University of Rome (Roma, Italy) \\ ${ }^{9}$ CNR-IOM Istituto Officina dei Materiali, Consiglio Nazionale delle Ricerche (Trieste, Italy) \\ ${ }^{10}$ INFN National Institute for Nuclear Physics (Firenze, Italy)
}

\begin{abstract}
A large repulsion between particles in a quantum system can lead to their localization, as it happens for the electrons in Mott insulating materials. This paradigm has recently branched out into a new quantum state, the orbital-selective Mott insulator, where electrons in some orbitals are predicted to localize, while others remain itinerant. We provide a direct experimental realization of this phenomenon, that we extend to a more general flavour-selective localization. By using an atom-based quantum simulator, we engineer SU(3) Fermi-Hubbard models breaking their symmetry via a tunable coupling between flavours, observing an enhancement of localization and the emergence of flavour-dependent correlations. Our realization of flavour-selective Mott physics opens the path to the quantum simulation of multicomponent materials, from superconductors to topological insulators.
\end{abstract}

Interactions shape the collective behavior of manyparticle quantum systems, leading to rich phase diagrams where conventional and novel phases can be induced by a controlled variation of external stimuli. The most direct example is perhaps the Mott insulator, where the large repulsion amongst particles leads to an insulating state despite the non-interacting system being a metal or a superfluid ${ }^{1}$. In solid-state physics the interest in Mott insulators is reinforced by the observation that the proximity to a Mott transition is a horn of plenty where a variety of novel and spectacular phases can be observed, high- $\mathrm{T}_{c}$ superconductivity $^{2,3}$ being only the tip of an iceberg ${ }^{4}$.

In recent years it has become clear that the standard $\mathrm{SU}(2)$ Fermi-Hubbard model is only a specific example, as many interesting materials require a description in terms of "multicomponent" Hubbard models, e.g. when the conduction electrons have an additional orbital degree of freedom. These systems are not merely more complicated, rather they host new phenomena, challenging the standard paradigm of Mott localization ${ }^{5}$. Indeed, when the symmetry between orbitals is broken, by some field or internal coupling, electrons in specific orbitals (or some combinations of them) can be Mott-localized while others remain itinerant, leading to surprising "orbital-selective Mott insulators" ${ }^{6}$.

Orbital-selective Mott physics has become a central concept for the description of a new class of high- $\mathrm{T}_{c}$ superconductors based on iron ${ }^{7}$, as it can describe the anomalies of the metallic state ${ }^{8,10}$ and the orbital character of superconductivity ${ }^{11}$ in those systems. However, a clean observation of selective Mott transitions is intrinsi- 58 cally hard in solid-state systems, because of the limited 59

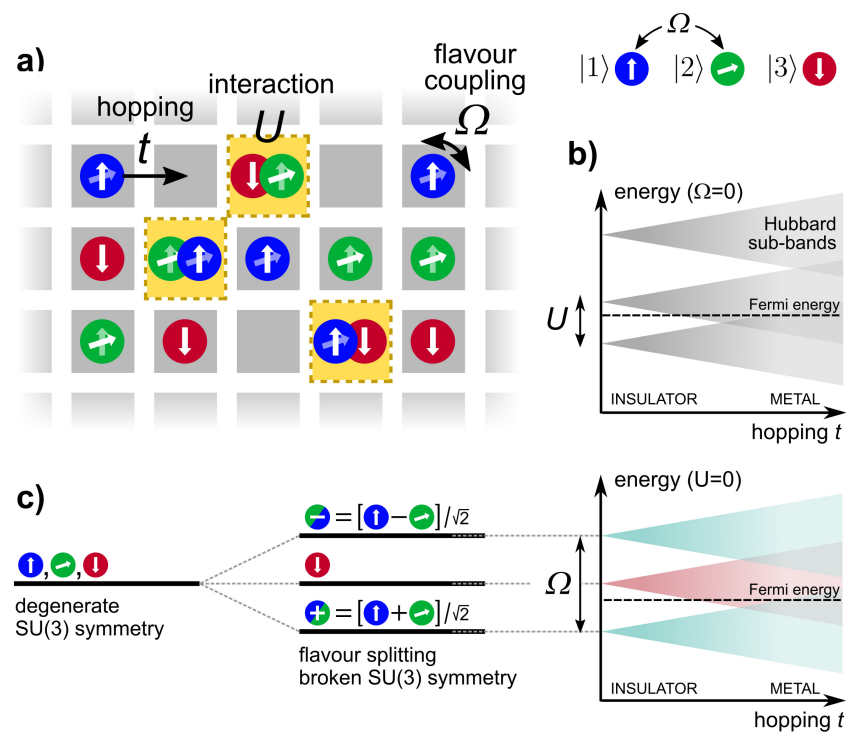

FIG. 1. Sketch of the physical system. a) We consider a system of repulsively $\mathrm{SU}(3)$-interacting fermions in a lattice. The global symmetry is broken by a coherent Rabi driving $\Omega$ between two internal states. b) In the absence of coupling, the system experiences a phase transition from an $\mathrm{SU}(3)$ metal to a Mott insulator as the hopping is reduced. c) The Rabi driving lifts the degeneracy between the states and, in a dressedbasis picture, causes them to acquire different energies. The competition with the hopping can drive a transition from a metal to an insulator already in the noninteracting case.

experimental control over the microscopic parameters and the orbital degree of freedom. The paradigm of selective 
a)

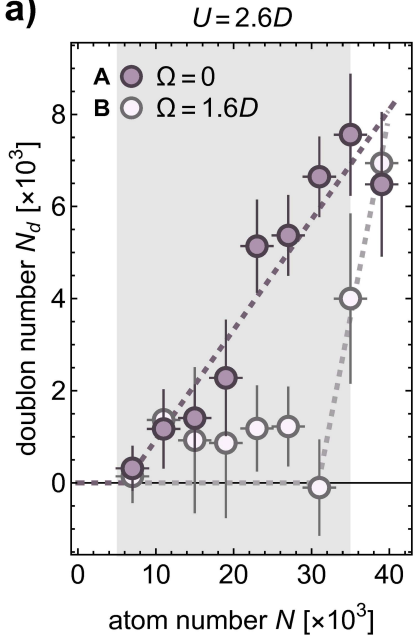

b)

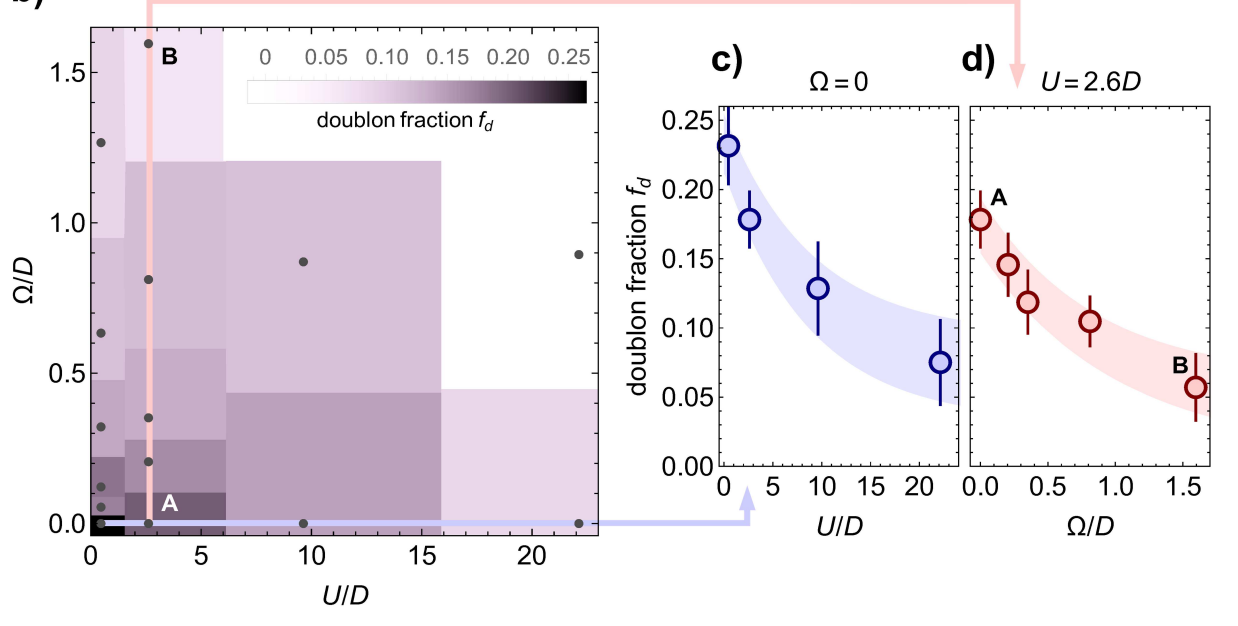

FIG. 2. Measurement of double occupancies. a) Number of atoms in doubly-occupied sites $N_{d}$ as a function of the total atom number $N$ for two different values of $\Omega$ and the same $U=2.6 D$. b) Average doublon fraction $f_{d}$ as a function of $U / D$ and $\Omega / D$. The actual measurements are marked by the points. c,d) Subsets of the data are shown for two different cross sections of the plot in b), i.e. for $\Omega=0$ (c) and for $U=2.6 D(\mathrm{~d})$. The measurements in b,c,d) are averages over an interval $N=(5 \ldots 35) \times 10^{3}$. Error bars in a,c,d) are obtained with a bootstrap analysis ${ }^{20}$. Dotted lines in a) are fits with a piecewise function (null + straight line), while color shades in $\mathrm{c}, \mathrm{d}$ ) are guides to the eye representing the experimental uncertainty.

Mott physics is itself far from being fully explored and has the potential to become a powerful framework to understand a variety of phenomena, from superconductivity to topological properties, in multicomponent quantum materials.

In this work we take a broader perspective and treat orbital-selective Mott physics as an example of the gen-91 eral concept of "flavour-selective Mott localization"12,92 which can be realized in a variety of multi-flavour sys- 93 tems, where the flavour can be the spin, the orbital or any 94 other quantum number. We realize a minimal instance of 95 this phenomenon by means of an atomic quantum simu-96 lator based on the optical manipulation of nuclear-spin ${ }_{97}$ mixtures of ultracold two-electron ${ }^{173} \mathrm{Yb}$ atoms. This ${ }_{98}$ platform allows the realization of multicomponent systems with global SU(N) interaction symmetry ${ }^{13,14}$, as in ${ }_{100}^{99}$ recent works reporting the realization of $\mathrm{SU}(\mathrm{N})$ quantum $_{101}$ wires $^{15}, \mathrm{SU}(\mathrm{N})$ Mott insulators ${ }^{16,17}$ and, more recently $\mathrm{SU}(\mathrm{N})$ quantum magnetism ${ }^{18}$. Here we introduce a novel ${ }_{103}$ approach to break the symmetry of SU(3) Fermi-Hubbard ${ }_{104}^{103}$ systems in a controlled way, which allows us to go beyond ${ }_{105}^{104}$ the investigations in the solid state and to observe di- ${ }^{105}$ rectly the two key signatures of selective-Mott physics ${ }^{12}{ }_{107}^{100}$ an overall enhancement of Mott localization and the onset of flavour-selective correlations.

The experiment is The experiment is performed with three-componentin ultracold ${ }^{173} \mathrm{Yb}$ Fermi gases with total atom number up ${ }_{112}$ to $N=4 \times 10^{4}$ and temperature $T \simeq 0.2 T_{F}$. The atoms113 are trapped in a cubic 3D optical lattice (lattice constant ${ }_{114}$ $d=\lambda / 2=380 \mathrm{~nm})$, which realizes the multi-flavour ${ }_{115}$ Hubbard Hamiltonian

$$
\begin{aligned}
\hat{H}= & -t \sum_{\langle i, j\rangle, \alpha}\left(\hat{c}_{\alpha i}^{\dagger} \hat{c}_{\alpha j}+\text { h.c. }\right)+U \sum_{i, \alpha, \beta \neq \alpha} \hat{n}_{\alpha i} \hat{n}_{\beta i}+\hat{V}_{T} \\
& +\frac{\Omega}{2} \sum_{i}\left(\hat{c}_{1 i}^{\dagger} \hat{c}_{2 i}+\text { h.c. }\right)
\end{aligned}
$$

where $\alpha, \beta \in\{1,2,3\}$ indicate the fermionic flavours (corresponding to nuclear spin states $m=+5 / 2,+1 / 2$ and $-5 / 2$, respectively), $t$ is the tunnelling energy between nearest-neighboring sites $\langle i, j\rangle, U$ is the onsite repulsion energy between two atoms of different flavours, and $\hat{V}_{T}=\kappa \sum_{i, \alpha} R_{i}^{2} \hat{n}_{\alpha i}$ describes the effects of a slowlyvarying harmonic trapping potential (where $R_{i}$ is the distance of site $i$ from the trap center). In the absence of the 4th term of Eq. (1), $\hat{H}$ has an intrinsic global $\mathrm{SU}(3)$ symmetry, which is ensured by the invariance of atom-atom interactions on the spin state and by the realization of spin-independent optical potentials, i.e. $U, t$ and $\kappa$ do not depend on $\alpha$. This symmetry is explicitly broken by the 4th term, which describes a coherent onsite coupling between flavours $|1\rangle$ and $|2\rangle$. This coupling is provided by a two-photon Raman process with Rabi frequency $\Omega / h^{20}$ (where $h$ is the Planck constant). At the single-particle level, this coupling lifts the degeneracy between the flavours, creating two dressed combinations $| \pm\rangle=(|1\rangle \pm|2\rangle) / \sqrt{2}$, energy-shifted from $|3\rangle$ by $\pm \Omega / 2$, as sketched in Fig. 1c.

We use an adiabatic preparation sequence to produce an equilibrium state of the atomic mixture in the optical lattice, with equal state populations $N_{1}=N_{2}=N_{3}=N / 3$ and in the presence of the coherent coupling $\Omega$ between states $|1\rangle$ and $|2\rangle^{20}$. In order to characterize the degree 

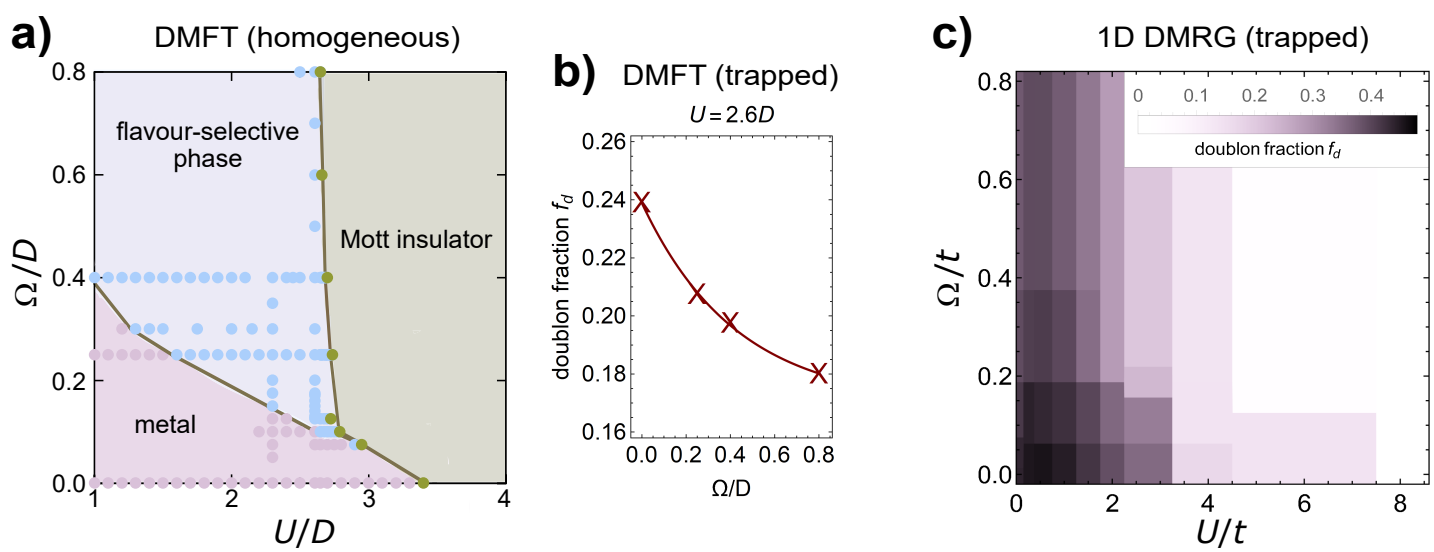

FIG. 3. Theoretical analysis. a) DMFT phase diagram for a homogeneous system at $T=0$ and $1 / 3$ filling. The points refer to different parameter runs. The boundary between the standard metal and the region of selective correlations marks a sharp crossover where the quasiparticle weight of the coupled flavours rapidly drops to a value smaller than 0.05 . b) Doublon fraction for the trapped system at $U=2.6 D$ and different $\Omega / D$, obtained from a LDA analysis of the homogeneous DMFT results with the experimental parameters. c) Doublon fraction obtained from DMRG for a 1D trapped system of $N=21$ particles at $T=0$.

of localization of the particles in the lattice, we measure 154 the number of atoms $N_{d}$ in doubly-occupied sites (called ${ }_{155}$ "doublons" in the following) with photoassociation spec $_{150}$ troscopy, as in previous experiments that demonstrated ${ }_{157}$ the onset of Mott localization for ultracold fermions ${ }^{16,19,20}{ }_{158}$ In Fig. 2a we report typical measurements of $N_{d}$ as $\mathrm{a}_{159}$ function of the total atom number $N$. In a harmonically $y_{160}$ trapped system, the rate of change of $N_{d}$ vs $N$ gives infor ${ }_{-161}$ mation on the core compressibility ${ }^{19,21}$. A vanishing value ${ }_{162}$ of $N_{d}$ over an extended range of $N$ signals the presence ${ }_{163}$ of an incompressible state with one atom per site in the $\mathrm{e}_{164}$ center of the trap (since adding particles does not lead ${ }_{165}$ to a proportional increase of doublons), while the critical ${ }_{160}$ $N$ above which $N_{d}$ then departs from zero can be con-167 nected to the magnitude of the energy gap protecting the ${ }_{168}$ localized phase. Fig. 2a shows two datasets for $U=2.6 D_{169}$ (where $D=6 t$ is the tunnelling energy times the lattice 170 coordination number), and two different Rabi couplings ${ }_{171}$ $\Omega=0$ and $\Omega=1.6 D$, respectively. The range of $N$ in the 172 figure spans a range of local fillings up to $\approx 0.8$ atoms/site ${ }_{173}$ per state (for the largest $N$ ) in the center of the trap $i_{174}$ the noninteracting case. The comparison between the two ${ }_{175}$ datasets clearly shows that the Rabi coupling $\Omega$ results 176 in an enhanced suppression of doublons, enlarging the ${ }_{177}$ region of $N$ where the incompressible state forms.

In the following we take the doublon fraction $f_{d}={ }_{179}$ $\left\langle N_{d} / N\right\rangle$, averaged over the $N$ interval marked by the gray 180 region in Fig. 2a, as an indicator of the degree of Mott181 localization of the system. The measured values of $f_{d^{182}}$ are shown as a function of $U$ and $\Omega$ in the diagram of ${ }_{183}$ Fig. 2b, clearly revealing the cooperative effect of Rabi 184 coupling and repulsive interactions driving the system ${ }_{185}$ towards a Mott-localized state. The same data are plotted ${ }_{186}$ with error bars in Figs. 2c,d along two different line cut\$187 of the diagram in Fig. 2b. Fig. 2c shows the effect 188 of an increasing $U$ in the transition towards an $\mathrm{SU}(3)_{189}$ Mott insulator for $\Omega=0$, while Fig. 2d shows a similar 190 localization effect induced by $\Omega$ at a fixed interaction strength $U=2.6 D$.

We now show the comparison of the experimental results with different theoretical analysis of the model in Eq. (1). Fig. 3a shows a zero-temperature phase diagram obtained from Dynamical Mean-Field Theory (DMFT) ${ }^{22}$ for the homogeneous system $\left(V_{T}=0\right)$ with a uniform $1 / 3$ filling (one atom per site) and equal number of particles for each flavour. The phase diagram clearly has the same shape of the experimental one, showing that the Hubbard $U$ and the coupling $\Omega$ cooperate in driving the system from a metallic phase to a more localized state. Between the standard metal and the Mott phase, we find a region where the degree of correlations (as measured by the quasiparticle weight) is strongly selective, the coupled flavours being much more localized than the uncoupled one. It is evident that both selective and global Mott localization occur at a smaller $U$ if $\Omega$ is included.

In order to connect this effect to the experimentally measured signal, in Fig. 3b we show the doublon fraction $f_{d}$ obtained from the homogeneous DMFT results after a local-density approximation (LDA) analysis, to take into account the effect of the harmonic trapping in $V_{T}$. The reduction of $f_{d}$ with increasing $\Omega$ is in agreement with the experimental observations reported in Fig. 2 . The lack of a quantitative matching with Fig. 2d can be attributed to imperfections in the initial state preparation and to the finite temperature of the experiment, resulting in an average entropy per particle $S / N \approx 2.5 k_{B}$, which is known ${ }^{21}$ to produce an effect on the double occupancies in a trapped system already at $\Omega=0$.

In Fig. $3 \mathrm{c}$ we finally show the result of a zerotemperature DMRG calculation of $f_{d}$ for a harmonically trapped $1 \mathrm{D}$ system ${ }^{20}$. Although a quantitative agreement with the experimental data should not be seeked (because of the different dimensionality and the finite temperature of the experimental realization), the overall behavior, i.e. 

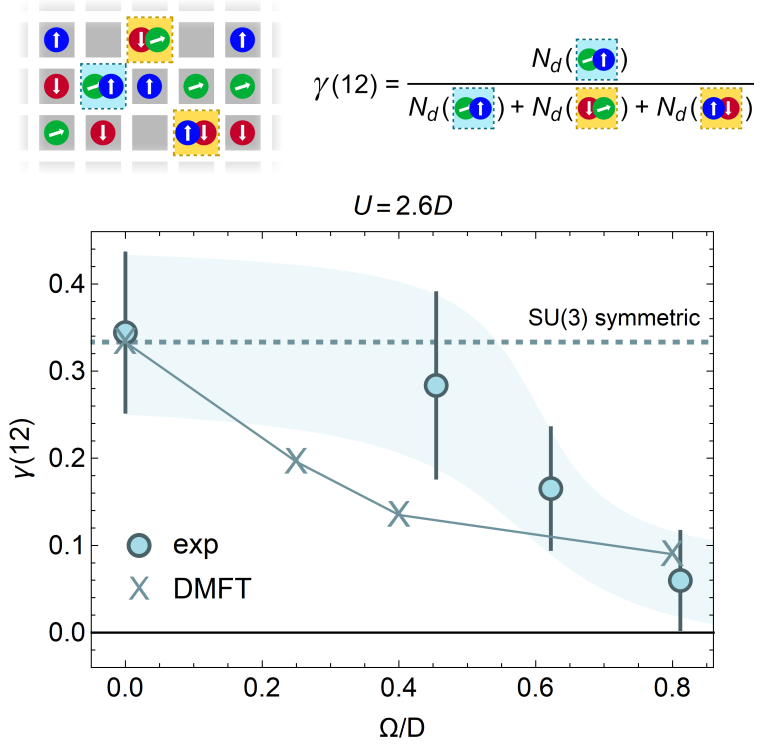

FIG. 4. Evidence of state-selective correlations. Experimental values of $\gamma(12)$ as a function of $\Omega / D$ for a fixed $U=2.6 D$. The circles are averages over an interval $N=$ $(5 \ldots 35) \times 10^{3}$, error bars are obtained from a bootstrap ${ }^{242}$ analysis $^{20}$, while the color shade is a guide to the eye. The ${ }^{243}$ crosses are obtained from a LDA analysis of the DMFT re- ${ }^{244}$ sultes. The dashed line shows the expected value for $\operatorname{an}^{245}$ SU(3)-symmetric system.

a reduction of doublons for increasing $U$ and $\Omega$, is $\operatorname{cor}^{249}$ rectly captured and agrees also with DMFT. This also250 indicates that the phenomena we are exploring are generic ${ }^{251}$ and qualitatively independent on the dimensionality. $\quad{ }^{252}$

In addition to the enhancement of Mott localization,253 the DMFT phase diagram of Fig. 3a shows that $\Omega$ is254 expected to result in a flavour-dependent localization of 255 the many-body system. The flavour-selective behavior250 can be experimentally detected by resolving the spin257 character of the doublons, i.e. counting how many atoms258 form doublons in each of the three pairs $|12\rangle,|23\rangle,|31\rangle_{259}$ by means of state-selective photoassociation at a high260 magnetic field ${ }^{20}$. In Fig. 4 we plot the quantity $\gamma(12)=261$ $N_{d}(12) / N_{d}$, where $N_{d}(12)$ is the number of atoms forming262 doublons in the $|12\rangle$ channel, as a function of $\Omega$ and fixed263 $U=2.6 D$. The measured value at $\Omega=0$ agrees with the264 expectation for an $\mathrm{SU}(3)$-symmetric system, for which265 $\gamma(12)=1 / 3$. As $\Omega$ is increased and the $\mathrm{SU}(3)$ symmetry is260 broken, $\gamma(12)$ diminishes, eventually approaching zero for 267 $\Omega \approx D$. The doublons acquire a strongly flavour-selective ${ }_{268}$ behavior.

This suppression of $|12\rangle$ doublons is triggered by the 270 polarization effect in the rotated $| \pm\rangle$ basis, which can 271 be understood, at a qualitative level, already from the 272 simplified, noninteracting case sketched in Fig. 1c: while 273 $|23\rangle$ and $|31\rangle$ doublons can be formed by two fermions in 274 the lowest single-particle states $|+\rangle$ and $|3\rangle,|12\rangle$ doublons275 can be formed only if the two fermions occupy states $|+\rangle_{276}$ and $|-\rangle$, therefore with an additional energy cost $\Omega / 2_{277}$
Interactions then increase this effect ${ }^{12}$, leading to strong flavour-selective results even for small values of $\Omega$.

The crosses in Fig. 4 are the result of a DMFT calculation in which the flavour populations are kept equal and the harmonic trapping $V_{T}$ has been taken into account in a LDA approach. The results of this numerical calculation are in good agreement with the experimental findings, with a larger degree of selectivity for the theoretical calculation. We argue that a better agreement could be seeked by including finite-temperature effects in the calculation: indeed, we expect the state-selective behavior to be reduced by the thermal occupation of higher-energy states, leading to an effective reduction of the polarization in the $|+\rangle,|-\rangle$ basis.

We note that the experimental observation of a finite number of $|12\rangle$ doublons at small, but finite $\Omega$ is an indication of the validity of the protocol used for the preparation of the atomic state, which is different for $\Omega=$ 0 and $\Omega>0^{20}$. We also note that, despite the polarization in the dressed $|+\rangle,|-\rangle$ basis discussed before, we have verified that the populations of the bare states $|1\rangle,|2\rangle,|3\rangle$ remain always equal under all the experimental conditions we have considered. This is indeed an important aspect of our experiment. If the populations of the bare states were not fixed (and in particular $N_{3}$ was left free to adapt), the dressed states would be populated according to the scheme in Fig. 1c, leading to $N_{1}=N_{2}>N_{3}$, which favours flavour-selective physics already in the non-interacting system. The experimental state preparation procedure counteracts the trivial differentiation between flavours by forcing an even occupation. Therefore, we conclude that the flavour selectivity we observed is essentially due to quantum correlations induced by interactions.

In the theoretical DMFT calculations, the equal population constraint is enforced including an external field $h^{20}$ that favours the occupation of $|3\rangle$ in such a way to match the experimental condition $N_{1}+N_{2}+N_{3}=N / 3$. Comparing with Ref. 12, where the populations were left free, we observe that the quantum correlations leading to the selective regime survive to the inclusion of the field $h$. In general terms, single-particle effects trigger flavour selectivity, but the inclusion of interactions strongly enhances the differentiation, turning a minor modulation of kinetic energy into a quantitative phenomenon which can also lead to a selective Mott transition ${ }^{24}$. This is a very general framework, underlying many investigations of multicomponent models.

Exploiting an idealized quantum simulator we have obtained a clear-cut evidence for correlation-induced flavourselective physics, where the $\mathrm{SU}(\mathrm{N})$ symmetry-breaking coupling $\Omega$ is only the trigger of a flavour-selective phenomenon which is fundamentally driven by correlation effects. Our first realization of multicomponent Hubbard physics with coherent internal couplings opens new paths for the quantum simulation of new classes of materials ranging from high-temperature superconductors to interacting topological insulators as described by the BernevigHughes-Zhang model ${ }^{23}$. As the coupling is realized with 
a nonzero momentum transfer (i.e. non collinear Raman 291 beams), a full range of possibilities will emerge, including 292 the study of magnetic crystals ${ }^{25}$, fractional quantum Hall ${ }_{293}$ states $^{26}$, and the effect of interactions of topological phase transitions ${ }^{27}$ and on the associated edge states ${ }^{28}$.

294

Acknowledgments. We acknowledge insightful dis-295 cussions with M. Dalmonte, D. Clément, F. Scazza and 296 financial support from projects TOPSIM ERC Consolida-297 tor Grant, TOPSPACE MIUR FARE project, QTFLAG 298 QuantERA ERA-NET Cofund in Quantum Technologies z29 MIUR PRIN project 2017E44HRF, MIUR PRIN project300 2015C5SEJJ, MIUR PRIN project 20172H2SC4_004,301 INFN FISh project. L.D.R was supported by the U.S 302
Department of Energy, Office of Science, Basic Energy Sciences, Division of Materials Sciences and Engineering under Grant No. DE-SC0019469.

Authors contribution. L.Fa., J.C. and M.C. conceived the experiments. D.T., L.F.L., L.Fr., D.B.O. and G.C. carried out the measurements. L.Fa., L.F.L., L.Fr. and D.T. analyzed the experimental results. K.B., L.D.R. and M.C. performed DMFT calculations. R.E.B. performed DMRG calculations. L.F.L. and L.Fa. performed exact diagonalization calculations. All authors contributed extensively to the discussion of the results and to the writing of the manuscript.
1 N. F. Mott, Proceedings of the Physical Society of London 348 Series A 62, 416 (1949).

2 P.W. Anderson, The resonating valence bond state ins $\mathrm{La} 2 \mathrm{CuO} 4$ and superconductivity, Science 235, 1196 (1987) 351

3 P. A. Lee, N. Nagaosa, and X.-G. Wen, Doping a Mott 352 insulator: Physics of high-temperature superconductivity,353 Rev. Mod. Phys. 78, 17 (2006).

4 S. Paschen and Q. Si, Nat. Rev. Phys. 3, 9 (2021)

5 A. Georges, L. de' Medici, J. Mravlje, Strong electronic corre-356 lations from Hund's coupling, Annual Reviews of Condensed 357 Matter Physics 4, 137 (2013)

6 M. Vojta, Orbital-selective Mott transitions: Heavy fermions and beyond, J. Low. Temp. Phys. 161, 203 (2010)

7 Y. Kamihara, T. Watanabe, M. Hirano, and H. Hosono, Iron-361 Based Layered Superconductor $\mathrm{La}\left[\mathrm{O}_{1-x} \mathrm{~F}_{x}\right] \mathrm{FeAs}(\mathrm{x}=0.05-362$ 0.12) with $\mathrm{T}_{c}=26 \mathrm{~K}$, Am. Chem. Soc. 130, 3296 (2008)

8 L. de' Medici, G. Giovannetti, and M. Capone, Selective 364 Mott Physics as a Key to Iron Superconductors, Phys. Rev 365 Lett. 112, 177001 (2014)

9 A. Kostin, P. O. Sprau, A. Kreisel, Yi Xue Chong, A. E ${ }_{367}$ Böhmer, P. C. Canfield, P. J. Hirschfeld, B. M. Andersen and ${ }_{368}$ J. C. Séamus Davis, Imaging orbital-selective quasiparticles 369 in the Hund's metal state of FeSe, Nature Materials 17, $869_{370}$ (2018)

10 M. Capone, Orbital-selective metals, Nature Materials 17 855 (2018)

11 P. O. Sprau, A. Kostin, A. Kreisel, A. E. Böhmer, V Taufour, P. C. Canfield, S. Mukherjee P. J. Hirschfeld, B 375 M. Andersen, and J. C. Séamus Davis, Discovery of orbital-376 selective Cooper pairing in FeSe, Science 357, 75 (2017) 377

12 L. Del Re and M. Capone, Selective insulators and anoma-378 lous responses in three-component fermionic gases with bro-379 ken SU(3) symmetry, Phys. Rev. A 98, 063628 (2018).

13 M. A. Cazalilla and A. M. Rey, Ultracold Fermi Gases with 381 Emergent SU(N) Symmetry, Rep. Prog. Phys. 77, 124401382 (2014).

14 A. V. Gorshkov, M. Hermele, V. Gurarie, C. Xu, P. S Julienne, J. Ye, P. Zoller, E. Demler, M. D. Lukin, and $\mathrm{d}_{385}$ A. M. Rey, Two-orbital SU(N) magnetism with ultracold 386 alkaline-earth atoms, Nature Phys. 6, 289 (2010).

15 G. Pagano, M. Mancini, G. Cappellini, P. Lombardi, F 388 Schäfer, H. Hu, X.-J. Liu, J. Catani, C. Sias, M. Inguscio, 389 and L. Fallani, A one-dimensional liquid of fermions with 390 tunable spin, Nature Phys. 10, 198-201 (2014).

16 S. Taie, R. Yamazaki, S. Sugawa, and Y. Takahashi, An 392
SU(6) Mott insulator of an atomic Fermi gas realized by large-spin Pomeranchuk cooling, Nature Phys. 8, 825-830 (2012).

17 C. Hofrichter, L. Riegger, F. Scazza, M. Höfer, D. Rio Fernandes, I.Bloch, and S. Fölling, Direct Probing of the Mott Crossover in the SU(N) Fermi-Hubbard Model, Phys. Rev. X 6, 021030 (2016).

${ }^{18}$ H. Ozawa, S. Taie, Y. Takasu, and Y. Takahashi, Antiferromagnetic Spin Correlation of SU(N) Fermi Gas in an Optical Superlattice, Phys. Rev. Lett. 121, 225303 (2018).

19 R. Jördens, N. Strohmaier, K. Günter, H. Moritz, and T. Esslinger, A Mott insulator of fermionic atoms in an optical lattice, Nature 455, 204-207 (2008).

20 See Supplementary Materials.

21 R. Jördens, L. Tarruell, D. Greif, T. Uehlinger, N. Strohmaier, H. Moritz, T. Esslinger, L. De Leo, C. Kollath, A. Georges, V. Scarola, L. Pollet, E. Burovski, E. Kozik, and M. Troyer, Quantitative Determination of Temperature in the Approach to Magnetic Order of Ultracold Fermions in an Optical Lattice, Phys. Rev. Lett. 104, 180401 (2010). 22 A. Georges, G. Kotliar, W. Krauth, and M. J. Rozenberg, Dynamical mean-field theory of strongly correlated fermion systems and the limit of infinite dimensions, Rev. Mod. Phys. 68, 13 (1996).

23 B. A. Bernevig, T. L. Hughes, and S.-C. Zhang, Quantum Spin Hall Effect and Topological Phase Transition in HgTe Quantum Wells, Science 314, 5806 (2006).

24 L. de' Medici, S. R. Hassan, M. Capone, and X. Dai, OrbitalSelective Mott Transition out of Band Degeneracy Lifting, Phys. Rev. Lett. 102, 126401 (2009).

25 S. Barbarino, L. Taddia, D. Rossini, L. Mazza, and R. Fazio, Magnetic crystals and helical liquids in alkaline-earth fermionic gases, Nat. Comm. 6, 8134 (2015).

26 M. Calvanese Strinati, E. Cornfeld, D. Rossini, S. Barbarino, M. Dalmonte, R. Fazio, E. Sela, and L. Mazza, Laughlin-like States in Bosonic and Fermionic Atomic Synthetic Ladders, Phys. Rev. X 7, 021033 (2017).

27 A. Amaricci, J. C. Budich, M. Capone, B. Trauzettel, and G. Sangiovanni, First-Order Character and Observable Signatures of Topological Quantum Phase Transitions, Phys. Rev. Lett. 114, 185701 (2015)

28 A. Amaricci, L. Privitera, F. Petocchi, M. Capone, G. Sangiovanni, and B. Trauzettel, Edge state reconstruction from strong correlations in quantum spin Hall insulators, Phys. Rev. B 95, 205120 (2017) 


\section{Supplementary Files}

This is a list of supplementary files associated with this preprint. Click to download.

- supplementarymaterials.pdf 